\title{
Development of a mobile magnetic resonance imaging system for outdoor tree measurements
}

\author{
Takeshi Kimura, ${ }^{1}$ Yuto Geya, ${ }^{1}$ Yasuhiko Terada,${ }^{1}$ Katsumi Kose,,${ }^{1, a)}$ Tomoyuki Haishi, ${ }^{2}$ \\ Hiroshi Gemma, ${ }^{3}$ and Yoshihiko Sekozawa ${ }^{3}$ \\ ${ }^{1}$ Institute of Applied Physics, University of Tsukuba, 1-1-1 Tennodai, Tsukuba, 3058573, Japan \\ ${ }^{2}$ MRTechnology Inc, 2-1-6 B5 Sengen, Tsukuba, 3050047, Japan \\ ${ }^{3}$ Agricultural and Forestry Research Center, University of Tsukuba, 1-1-1 Tennodai, Tsukuba, 3058577, Japan
}

(Received 24 December 2010; accepted 18 April 2011; published online 17 May 2011)

\begin{abstract}
By combining a $0.3 \mathrm{~T}$ permanent magnet with flexible rotation and translation mechanism, a probe with a local electromagnetic shielding, several electrical units, a mobile lift, and an electric wagon, a mobile magnetic resonance imaging (MRI) system was developed for outdoor tree measurements. 2D cross-sectional images of normal and diseased branches of a pear tree were acquired for measurements of $T_{1}, T_{2}$, proton density, and apparent diffusion constant (ADC). The ADC map clearly differentiated diseased from normal branches. A whole-day measurement of the ADC map demonstrated that microscopic water flow in the normal branch changed proportionally with solar radiation. Therefore, we have concluded that our mobile MRI system is a powerful tool for studies of plants in outdoor environments. (0) 2011 American Institute of Physics. [doi:10.1063/1.3589854]
\end{abstract}

\section{INTRODUCTION}

Magnetic resonance imaging (MRI) is a powerful noninvasive imaging tool for living systems. Although the most dominant MRI application is human medical diagnosis, plant measurements are also an important area for MRI applications. ${ }^{1-6}$

Until now, almost all MRI measurements of plants have been performed in laboratories using plants in pots or vessels. There are, of course, some exceptional studies in which plants were measured out of laboratories using some portable MRI systems. In 2000, Rokitta and his colleagues reported a portable MRI system for plants using a $0.47 \mathrm{~T}$ permanent magnet that can be used in a greenhouse and demonstrated several cross-sectional images of intact plants. ${ }^{7}$ Their system was very light (about $60 \mathrm{~kg}$ ), but the imaging area was limited (12 mm diameter sphere) and magnetic field drift, which was mainly caused by changes in air temperature, was not compensated for during the long measurement time. In 2006, Okada and her colleagues reported the first outdoor MRI measurements of a natural maple tree using a portable MRI system with a $0.3 \mathrm{~T}, 80 \mathrm{~mm}$ gap permanent magnet. ${ }^{8}$ However, because the flexibility of the MRI probe was limited, only a vertically straight tree was used in the experiments.

Although physiologically controlled MRI plant studies should be performed in laboratories, natural phenomena such as plant diseases and damage by insects should be investigated in outdoor environments using portable measurement systems. However, if one wants to measure plants in farms or forests for long-term or routine MRI measurements, there are many problems to be overcome.

The first problem is portability of the MRI system. This is because the topography of such measurement sites is gen-

a)Electronic mail: kose@bk.tsukuba.ac.jp. Tel: +81-29-853-5214. FAX: +81-29-853-5205. erally rough and soft, and so it is difficult to move heavy MRI systems to the plants to be measured. It is also difficult or time consuming to carry disassembled MRI units to the measurement sites, to set up the system in such outdoor environments and to reproduce the good performance achieved in a laboratory. One solution to this problem is to construct a portable MRI system such that the whole assembly can be moved directly from the laboratory to the measurement sites.

The second problem, not previously reported, is that it is difficult to set up the magnet to fit the subject plants because plant stems and tree branches have varied and irregular diameters, shapes, spatial locations, and orientations. To solve this problem, the magnet must be designed to have flexible positioning and orientation.

The third problem is temperature drift of the magnet. If one wants to perform outdoor measurements of plants, permanent magnets are the first choice as a magnet. However, because temperature drift of the magnetic field of the permanent magnet is generally very large, e.g. about $-1000 \mathrm{ppm} /$ degree for magnets using $\mathrm{NdFeB}$ material, compensation or correction techniques for the temperature drift should be developed. In addition, because temperature variation in the outdoor environments is usually much larger than that in standard laboratories, this problem becomes very serious for outdoor measurements compared with laboratory measurements.

The fourth problem is the external electromagnetic noise coming into the image area. Although external noise such as radio waves can be shielded using a shielding room in laboratories, it is difficult to construct such a shielding room in outdoor environments while keeping the imaging object in a natural condition. Moreover, because the plant's water is generally a good antenna for external radio waves, some electromagnetic shielding techniques should be developed.

In this study, we overcame the problems described above for outdoor in situ tree measurements by developing an electrically motorized mobile MRI system, and we succeeded in 


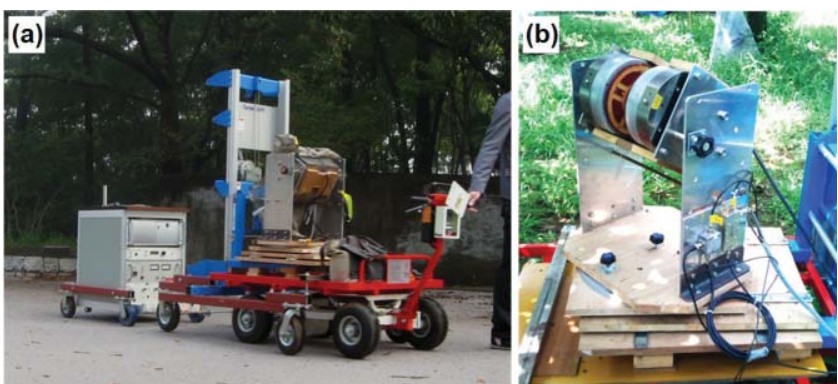

FIG. 1. (Color online) (a) Overview of the electrically motorized mobile MRI system using a permanent magnet. (b) Close view of the permanent magnet with two rotation axes and two horizontally sliding tables. The rotation axes are vertical and horizontal axes.

a whole-day outdoor MRI measurement of branches of a pear tree at a fruit farm.

\section{MATERIALS AND METHODS}

\section{A. Mobile MRI system}

Figure 1(a) shows an overview of the mobile MRI system developed in this study. The system consists of an electrically motorized wagon, a mobile lift, a permanent magnet, a gradient coil set, an RF probe, and an MRI console.

The wagon (CB-02, Yamaichi-Seikou, Suzaka, Japan) is driven by an electric motor supplied by a Ni-H battery controlled by a power assist system. The specification is as follows: pallet size, $1090 \mathrm{~mm} \times 780 \mathrm{~mm}$; weight, $55 \mathrm{~kg}$; maximum bearing weight, $200 \mathrm{~kg}$; running distance per charge, $6.4 \mathrm{~km}$. After the original wheels of the mobile lift (GL-4, Genie Industries, WA, USA) were replaced with wheels with larger tires, the lift was mounted on and connected to the electric wagon using several nuts and bolts to move it together with the wagon using eight tires as shown in the figure. The maximum height and bearing weight of the lift fork is $140 \mathrm{~cm}$ and $225 \mathrm{~kg}$, respectively.

The specification of the permanent magnet (Neomax Engineering, Tokyo, Japan) is as follows: magnetic field strength, $0.3 \mathrm{~T}$; gap width, $80 \mathrm{~mm}$; homogeneity, $60 \mathrm{ppm}$ over $30 \mathrm{~mm}$ diameter spherical volume; weight, $57 \mathrm{~kg}$. The permanent magnet was mounted on a turntable with two rotation axes and two horizontal sliding tables as shown in Fig. 1(b). The spatial orientation and horizontal position can be flexibly fixed to fit any plant stem or tree branch of less than $30 \mathrm{~mm}$ diameter. In addition, because the table was mounted on the fork of the lift, the magnet center can be placed to any height of the object up to about $160 \mathrm{~cm}$ from the ground.

The RF coil was wound directly around a pear tree branch using a spongy sheet and a $1.5 \mathrm{~mm}$ diameter polyurethane coated $\mathrm{Cu}$ wire [Fig. 2(a)]. The RF coil was about $20 \mathrm{~mm}$ in diameter with eight turns. It was placed in an RF shielded box made of 4-mm-thick acrylic plates and 0.1-mm-thick $\mathrm{Cu}$ foil attached to the faces of the acrylic plates [Fig. 2(b)]. The RF shielded box and the tree branch were electromagnetically shielded together using a shielding cloth [Fig. 2(c)]. This signal detection unit was installed in the gap of the permanent magnet by attaching the permanent magnet that has two rota-
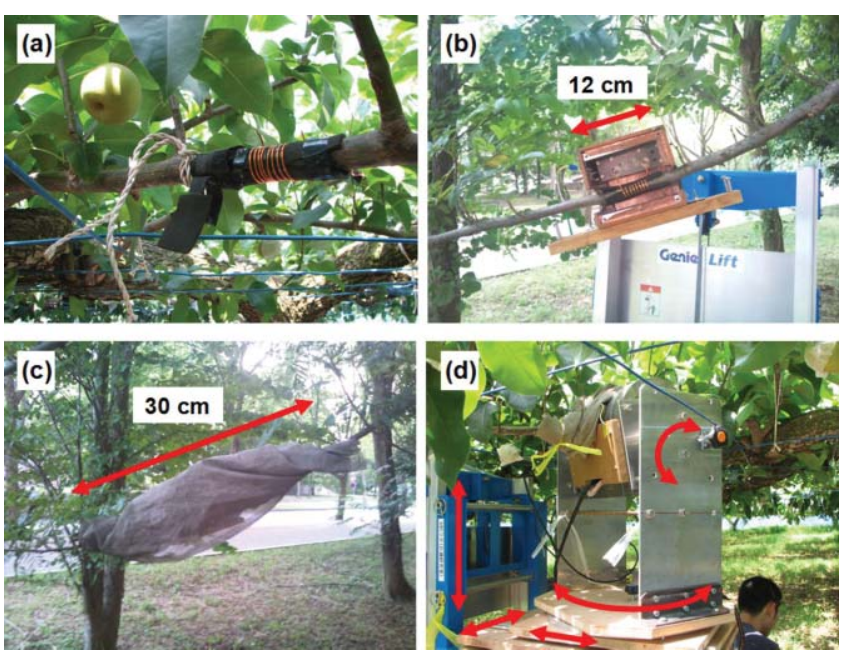

FIG. 2. (Color online) (a) RF coil wound around a branch of a pear tree. (b) Open view of the RF probe. (c) Electromagnetically shielded RF probe with a shielding cloth wound over the RF probe. (d) Setup of the RF probe in the gap of the permanent magnet. The magnet has two rotation axes and three translational mechanisms.

tion axes and vertical and horizontal translational mechanisms [Fig. 2(d)].

The gradient coil set consists of planar transverse (Gx and $\mathrm{Gy}$ ) and axial (Gz) gradient coils with a conventional design. The efficiencies of the gradient coils are 22, 8.0, and $7.3 \mathrm{mT} / \mathrm{m} / \mathrm{A}$ for $\mathrm{Gx}, \mathrm{Gy}$, and $\mathrm{Gz}$ gradient coils, respectively. The efficiency of the Gx coil was enhanced to measure apparent diffusion constant (ADC) along this direction.

The MRI console consists of an industrial PC with a Pentium 4 processor $(2.8 \mathrm{GHz}$ clock frequency) and a $128 \mathrm{~GB}$ solid state disc drive, an MRI transceiver (DTRX-4, MRTechnology, Tsukuba, Japan), a transmitter (T145-5057A, 100 W, 5-80 MHz, Thumway, Fuji, Japan), and a three-channel gradient driver $( \pm 20 \mathrm{~V}, \pm 10 \mathrm{~A}$, Jyonan Electric Laboratory, Tsukuba, Japan). These units were installed in a 19-inch rack $(54 \mathrm{~cm}(\mathrm{~W}) \times 77 \mathrm{~cm}(\mathrm{H}) \times 60 \mathrm{~cm}(\mathrm{D}))$ and mounted on a carrier with four tires as shown in Fig. 1(a). Total weight of the MRI console including the carrier was about $100 \mathrm{~kg}$. A $100 \mathrm{~V} \mathrm{AC}$ single-phase power source is needed for the console whose maximum power consumption was about $600 \mathrm{~W}$ for imaging experiments. The details of the MRI console are described elsewhere. ${ }^{9}$

\section{B. Pear tree}

Two branches of a Japanese pear tree (Pyrus pyrifolia Nakai, Kosui, about 35 years old) in the Agricultural and Forestry Research Center of the University of Tsukuba were used for the MRI measurements. One branch was normal and the other had dwarf disease, which causes leaves and fruit to become small compared with those on normal healthy branches. The disease has become a serious problem and it is frequently observed in Japanese fruit farms. Because both branches were located about $1.6 \mathrm{~m}$ high, the magnet was raised to the top position of the lift for the measurements. 


\section{Experiments}

We performed the following four experiments.

The first experiment was performed to develop an RF shielding method to reject external electromagnetic noise coming into the image bandwidth. To measure the shielding efficiency of the shielding cloth, cross-sectional images of a normal branch were measured using a 2D spin-echo sequence with and without the cloth.

The second experiment involved measurements of NMR parameters $\left(T_{1}, T_{2}\right.$, and proton density) in the cross sections of the two branches. For this experiment, 2D spinecho sequences with TR (repetition time)/TE (echo time) of $2000 \mathrm{~ms} / 10 \mathrm{~ms}, 2000 \mathrm{~ms} / 50 \mathrm{~ms}, 1000 \mathrm{~ms} / 10 \mathrm{~ms}$, and $500 \mathrm{~ms} / 10 \mathrm{~ms}$ were used.

The third experiment involved ADC measurements to visualize microscopic water flow in the cross sections of the two branches. ${ }^{10}$ For this experiment, conventional diffusion weighted 2D spin-echo sequences using a motion probing gradient (MPG) with TR/TE $=800 \mathrm{~ms} / 22$ ms were used. ${ }^{11}$ Parameters for the MPG were: $\Delta=12 \mathrm{~ms}, \delta=6 \mathrm{~ms}$, and gradient field strength $=0,55,83$, and $110 \mathrm{mT} / \mathrm{m}$, where $\delta$ and $\Delta$ are the duration of each MPG and the separation time between the MPG pair, respectively. Relative $b$ values for these pulse sequences were $0,78,177$, and $314 \mathrm{~s} / \mathrm{mm}^{2}$.

The fourth experiment was a whole-day ADC measurement performed to confirm validity of the measurements and the system performance. An ADC map of the cross section of the normal branch was measured every $2 \mathrm{~h}$ throughout a sunny day in August 2010. Solar radiation was measured using a solarimeter (IKS-37, Koito Industries, Yokohama, Japan) throughout the day.

For all the 2D spin echo image measurements described above, slice thickness, image matrix, and field of view (FOV) were $10 \mathrm{~mm}, 128 \times 128$, and $25.6 \mathrm{~mm} \times 25.6 \mathrm{~mm}$, respectively. All of the image measurements were performed in the single slice mode. Although the ratio of the slice thickness $(10 \mathrm{~mm})$ to the in-plane resolution $(0.2 \mathrm{~mm} \times 0.2 \mathrm{~mm})$ may seem unusual, we used straight part of the branches as much as possible to achieve high spatial resolution and SNR at the same time. There were four NMR signal accumulations (NEX), except in the RF shielding experiment where $\mathrm{NEX}=1$.

Because measurement time for one cross section was around $10 \mathrm{~min}$ for every measurement and the temperature drift of the magnetic field was serious, an internal NMR lock sequence ${ }^{12}$ was applied at the beginning of every phaseencoding step. As the result, the image measurement time became approximately doubled comparing with that without the NMR lock sequence. For example, the image measurement time for ADC measurements was nominally about $6.8 \mathrm{~min}$ $(\mathrm{TR}=800 \mathrm{~ms}$, image matrix $=128 \times 128, \mathrm{NEX}=4)$, but actually about $12 \mathrm{~min}$.

\section{RESULTS}

\section{A. Mobile performance of the MRI system}

Because the walking distance between the fruit farm and the authors' laboratory was about $1.5 \mathrm{~km}$, it took about 15

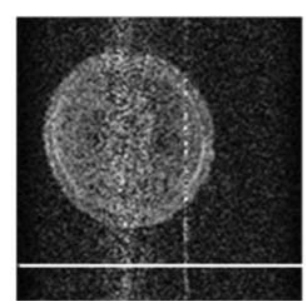

(a)

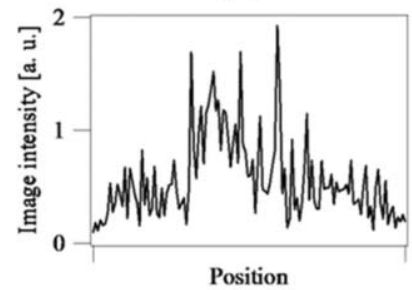

(c)

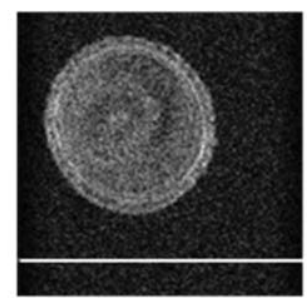

(b)

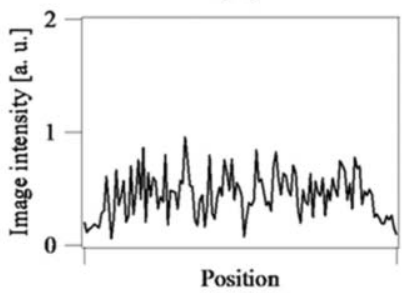

(d)
FIG. 3. Cross sectional MR images and profiles of a branch of a pear tree. (a) and (c) : No RF shielding. (b) and (d) : RF shielding with the shielding cloth.

min to move the MRI system from the laboratory to the measurement sites. Typical setup time for one branch was about 30 min including RF coil winding and probe tuning. Therefore, measurements of normal and diseased branches could be performed on the same day. In the fruit farm, although the ground was very rough and soft, the mobile MRI had no trouble with its transportation.

\section{B. RF shielding effect}

Figure 3 shows cross sectional images of the normal branch of the pear tree measured without shielding [Fig. 3(a) and 3(c)] and with the shielding cloth [Fig. 3(b) and 3(d)]. The images and profiles clearly show that the external noise was drastically reduced by RF shielding. The external noise rejection ratio was evaluated at more than $20 \mathrm{~dB}$, because background thermal noise originating mainly from the RF coil was present.

\section{NMR parameter mapping}

Figure 4 shows the distribution of $\mathrm{T}_{1}, \mathrm{~T}_{2}$, and proton density in the cross sections of the normal and diseased branches calculated from 2D spin echo images. These images clearly show several interesting properties of the normal and diseased branches. First, the density of mobile water protons is high around the xylem located close to the external surface of the branches, as is shown in Fig. 4(b) and 4(d). Second, the normal branch shows clear localization of mobile water protons with a longer $T_{1}$ value ( $\left.>600 \mathrm{~ms}\right)$, whereas the diseased branch has no such clear distribution in the $T_{1}$ map as shown in Fig. 4(a) and 4(c).

\section{ADC measurements}

Figure 5 shows diffusion-weighted and ADC images in the cross sections of normal and diseased branches. Relative $b$ 

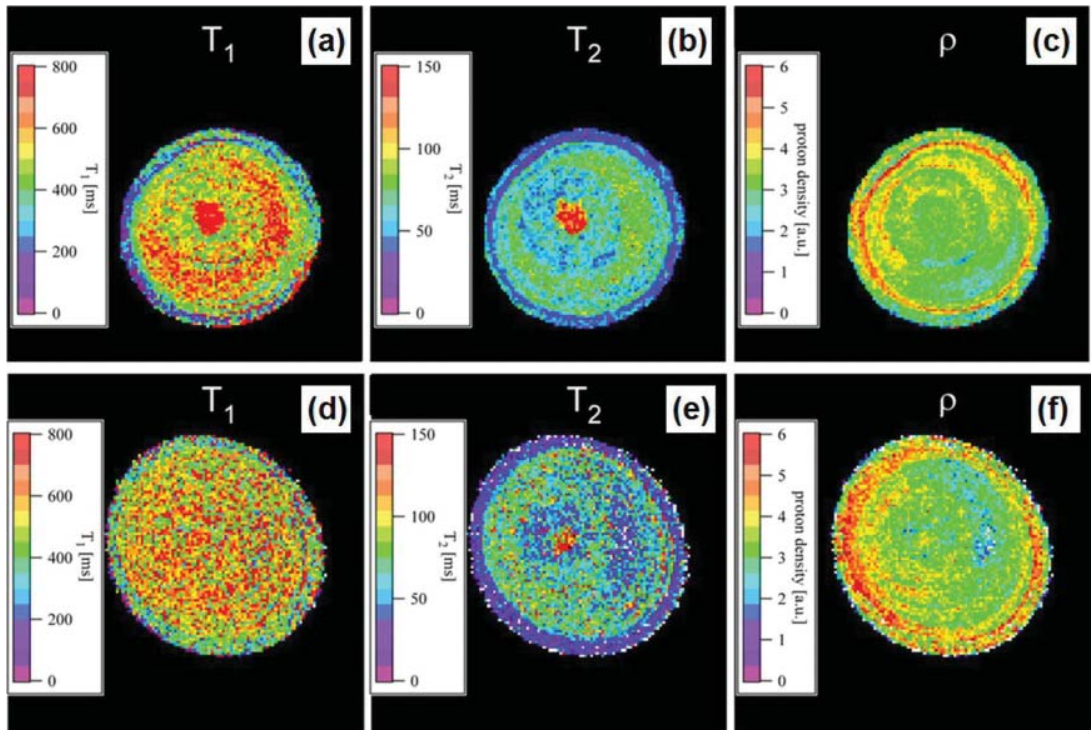

FIG. 4. (Color online) Computed $\mathrm{T}_{1}, \mathrm{~T}_{2}$, and proton density images of branches of a pear tree. (a)-(c) Normal branch. (d)-(f) Diseased branch.

values are $0 \mathrm{~s} / \mathrm{mm}^{2}$ in Fig. 5(a) and 5(d) and $314 \mathrm{~s} / \mathrm{mm}^{2}$ in Fig. 5(b) and 5(e). As shown in the ADC maps, while the normal branch clearly shows a definite high ADC area, the diseased branch has no definite high ADC area. This difference clearly shows that water is well transported in the sapwood area of the normal branch, but the water transport function is degraded or destroyed in the diseased branch. Although the $\mathrm{T}_{1}$ images show a similar property, the ADC maps show the difference much more clearly.

\section{E. Whole-day measurement}

Figure 6 shows a whole-day variation of ADC in the sapwood and heartwood of the normal branch and solar radiation measured every $2 \mathrm{~h}$ on a sunny day in August 2010. We can clearly see that ADC in the sapwood changed proportionally with the solar radiation but the ADC in the heartwood did not. This result clearly demonstrates that our system can detect the water transport in the pear tree branch even during long-term measurement.

\section{DISCUSSION}

Until now, several portable MRI systems have been reported. ${ }^{7,13-15}$ Because those systems are supposed to be used in indoor environments such as hospitals, their wheels were designed to be used on smooth floors and to be moved manually. However, this design is not suitable for outdoor field measurements.

In this study, we used large, robust wheels and an electrical motor driving system to construct the mobile MRI system for outdoor experiments. As far as we know, this
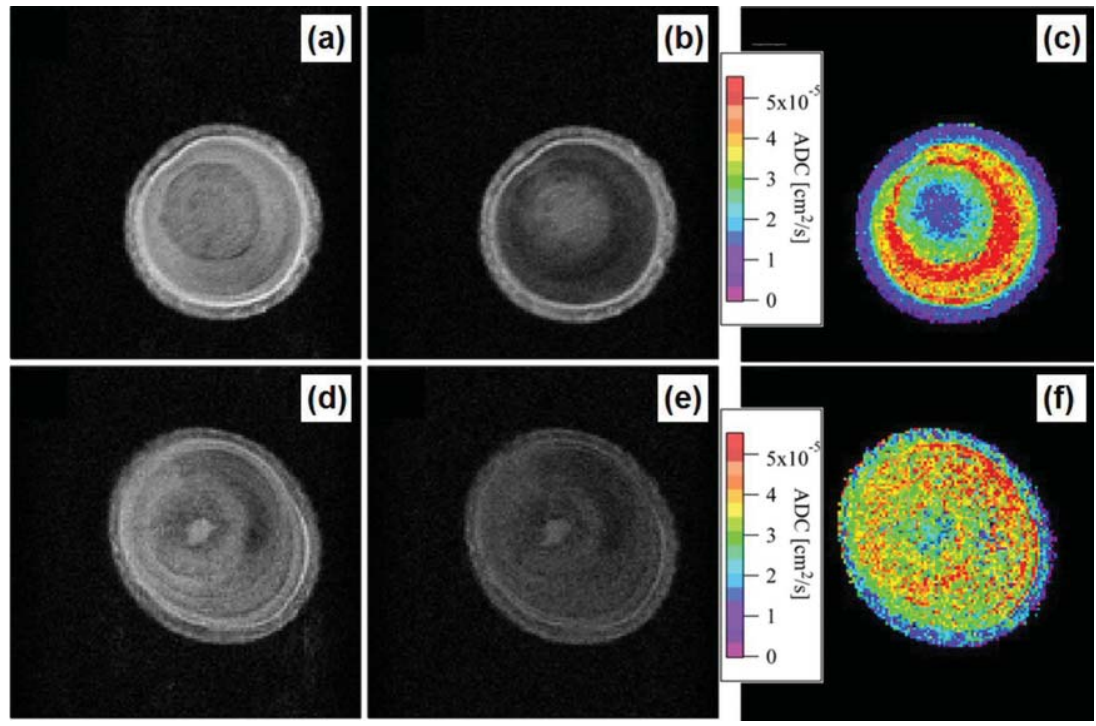

FIG. 5. (Color online) Diffusion weighted and ADC images of normal (a)-(c) and diseased (d)-(f) branches. (a) and (d) b $=0$ s/mm ${ }^{2}$. (b) and (e) $\mathrm{b}=314 \mathrm{~s} / \mathrm{mm}^{2}$. (c) and (f) ADC images. 


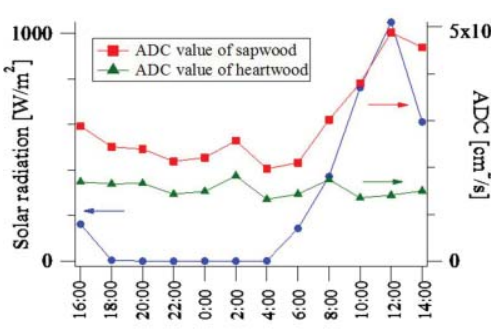

(a)

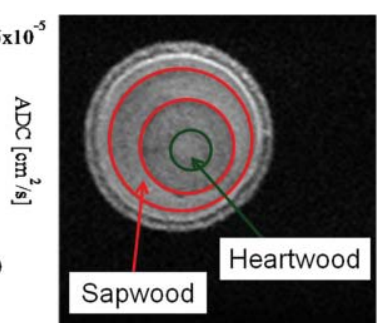

(b)
FIG. 6. (Color online) Whole day variation of solar radiation (closed circle) and ADC measured in two regions of the normal branch. The square and triangle show ADC in the sapwood and heartwood.

is the first system that includes a motorized driving system with a portable MRI system. In our system, the driving system and the rotation and sliding mechanism of the permanent magnet were essential for outdoor measurements of trees.

As shown in Fig. 3, we achieved more than $20 \mathrm{~dB}$ RF noise rejection ratio and succeeded in rejecting the external noise effect. However, if the external noise would be more intense, the RF noise rejection ratio should be more improved using additional conducting cloths or other flexible conductors such as $\mathrm{Cu}$ foil.

As shown in Figures 3-5, image artifacts caused by the magnetic field drift were not observed. This is because the NMR lock interval was so short (less than $10 \mathrm{~s}$ ) that the Larmor frequency drift $(300 \mathrm{~Hz} / \mathrm{min}$ or $50 \mathrm{~Hz} / 10 \mathrm{~s}$ at most in the daytime) was less than the pixel bandwidth $(195 \mathrm{~Hz})$. However, because the throughput for the image measurements was limited for this approach, temperature control of the permanent magnet is desirable for future developments.

As shown in Sec. III, the branch with dwarf disease was clearly differentiated from the normal branch using an ADC map. This result suggests that the measurement of the ADC with our MRI system can be a powerful tool for further clarifying the mechanism of dwarf disease in pear trees.

\section{CONCLUSION}

We have developed an electrically motorized mobile MRI system for measurements of pear tree branches to study the dwarf disease of pear trees. The ADC map clearly differentiated the diseased branch from the normal branch. A wholeday measurement of the ADC map demonstrated that the microscopic water flow in the branch varied proportionally with the solar radiation. Therefore, we have concluded that our mobile MRI system is a powerful tool for studies of plants in outdoor environments.

${ }^{1}$ T. W. J. Scheenen, D.van Dusschoten, P. A. de Jager, and H. Van As, J. Exp. Bot. 51, 1751 (2000).

${ }^{2}$ W. Köckenberger, J. Exp. Bot. 52, 641 (2001).

${ }^{3}$ N. M. Homan, C. W. Windt, F. J. Vergeldt, E. Gerkema, and H. Van As, Appl. Magn. Reson. 32, 157 (2007).

${ }^{4}$ K. Kuroda, Y. Kanbara, T. Inoue, and A. Ogawa, IAWA J. 27, 3 (2006).

${ }^{5}$ H. Van As, J. Exp. Bot. 58, 743 (2007).

${ }^{6}$ S. Utsuzawa, K. Fukuda, and D. Sakaue, Phytopathology, 95, 737 (2005).

${ }^{7}$ M. Rokitta, E. Rommel, U. Zimmermann, and A. Haase, Rev. Sci. Instrum. 71, 4257 (2000).

${ }^{8}$ F. Okada, S. Handa, S. Tomiha, K. Ohya, K. Kose, T. Haishi, S. Utsuzawa, and K. Togashi, paper presented at the 6th Colloquium on Mobile Magnetic Resonance, Aachen, Germany, 6-8 September 2006.

${ }^{9} \mathrm{~K}$. Kose, in NMR Imaging in Chemical Engineering, edited by S. Stapf, and S.-I. Han (Wiley, New York, 2006), p. 77.

${ }^{10}$ D. Le Bihan, E. Breton, D. Lallemand, P. Grenier, E. Cabanis, and M. Laval-Jeantet, Radiology 161, 401 (1986).

${ }^{11}$ E. Stejskal and J. Tanner, J. Chem. Phys. 42, 288 (1965).

${ }^{12}$ T. Haishi, T. Uematsu, Y. Matsuda, and K. Kose, Magn. Reson. Imaging 19, 875 (2001).

${ }^{13}$ G. Gold, D. Theodorou, T. Blair, G. Garcia, C. Crowley, F. Rose, D. Trudell1, and D. Resnik, 6th ISMRM abstract book (ISMRM, Sydney, 1998), p. 2039.

${ }^{14} \mathrm{~K}$. Kose, paper presented at the 5th International Conference on Magnetic Resonance Microscopy, Heidelberg, Germany, 4-9 September 1999.

${ }^{15}$ N. Iita, S. Handa, S. Tomiha, and K. Kose, Magn. Res. Med. 57, 272, (2007). 